\title{
Extra Nodal Primary Diffuse Large B-Cell Lymphoma of Thyroid : A Review of Literature
}

\author{
Anand Kalia, Sudhamani S*., Anita Sharan, Surekha Bhalekar and Prakash Roplekar \\ Department of Pathology, Dr. D. Y. Patil Medical College, Nerul, Navi Mumbai. India
}

\begin{abstract}
Primary Thyroid Lymphoma (PTL) involves less than $5 \%$ of all thyroid malignancies. It mostly comes into notice later in life of which Diffuse Large B-Cell Lymphoma (DLBCL) is the commonest type. Though the primary site of lymphoma is Lymph Node, DLBCL involving thyroid primarily and later spreading to Lymph Nodes and other sites is also known. This tumor causes rapid progression of the thyroid mass which can compress the trachea and may even present as an acute emergency. Therefore, it is important to recognize this entity as the treatment and progression differs from other thyroid malignancies

We are presenting the case of Extra Nodal Primary DLBCL of Thyroid gland in a 55 years old female who presented with thyroid swelling along with multiple Cervical Lymph Nodes which on Histopathology and subsequent Immunohistochemistry was confirmed as DLBCL of primary thyroid gland with regional and distant metastases.
\end{abstract}

Keywords: Thyroid, Lymphoma, Primary, Diffuse Large B-cell Lymphoma, DLBCL, Primary Thyroid Lymphoma

\section{Introduction}

Primary thyroid lymphoma (PTL) is a rare malignant tumor, defined as lymphoma involving only the thyroid gland or the thyroid and regional lymph nodes. It forms approximately $2 \%$ of all extranodal lymphomas commonly seen in spleen, thymus, Waldeyer's ring, etc. ${ }^{[1]}$

Most PTLs are of B-cell origin Non Hodgkin Lymphoma (NHL) and DLBCL comprises 50-70\% of these. Most of them develop in the background of Hashimoto's thyroiditis. [2]

PTL are amenable to treatment if recognized early. ${ }^{[1]}$ Therefore, it is important to recognize this rare entity as diagnosis requires careful histopathological examination and confirmation by Immunohistochemistry (IHC).

\section{Case report}

A 55 years old female patient came with complaint of swelling over neck and cervical region since 3 months. The swelling was gradually increasing in size and associated with muscular cramps and shooting pain over right shoulder and arm region. Occasional episodes of difficulty in breathing were also there. Patient was a known case of Diabetes Mellitus, Hypertension and Hypothyroidism on treatment. Thyroid Function Tests showed increased levels of Thyroid Stimulating Hormone. Serum calcitonin was under normal limits.

Fine Needle aspiration Cytology (FNAC) of thyroid showed features of high grade malignancy. Differential diagnosis of anaplastic carcinoma of thyroid and soft tissue sarcoma locally infiltrating into the thyroid was considered. Lymph Node FNAC \& biopsy was done which showed the features of metastasis of high grade malignancy. Patient underwent total thyroidectomy with radical neck dissection.

Grossly, we received both thyroid lobes and isthmus separately along with bilateral cervical lymph nodes. Right lobe measured $9.5 \times 5 \times 4.5 \mathrm{~cm}$ and left lobe measured 6.5 x 4 × $2.5 \mathrm{~cm}$. (Figure 1)

On serial sectioning, grey-white homogenous solid tumour involving the entire thyroid was noted.

Microscopically, both thyroid lobes and isthmus showed similar histomorphology. Monotonous population of atypical lymphoid cells were seen arranged in diffuse pattern. Individual tumor cells were large, round to polygonal, having irregular cell membrane, vesicular nuclei and prominent nucleoli with moderately abundant amphophilic cytoplasm. Focal areas of necrosis and haemorrhage were noted. Surrounding thyroid parenchyma showed features of Hashimoto's thyroiditis (HT). The surgical margins were free from the tumor. The cervical lymph nodes sent separately were free from tumor. Histopathological diagnosis of NHL was given with differential diagnosis of Anaplastic Carcinoma. (Figure 2).

IHC of the tumor showed strong positivity for CD20 and BCL 2 while Ki67 was $40 \%$ in the highest proliferative area. The final diagnosis of Diffuse Large B-Cell Lymphoma was made based on these findings. Whole body PET scan was later done and showed the evidence of metabolically active 
lymphadenopathy both above and below diaphragm and metabolically active splenic lesions (stage IIIs). Also mild FDG uptake was noted in bone marrow. Patient was put on chemotherapy treatment and doing well on subsequent follow-up of one year.
The chemotherapy regimen used was RCHOP (Rituximab, Cyclophosphamide, Doxorubicin, Vincristine, Prednisone) and had taken six cycles with an interval of three weeks between each cycle.

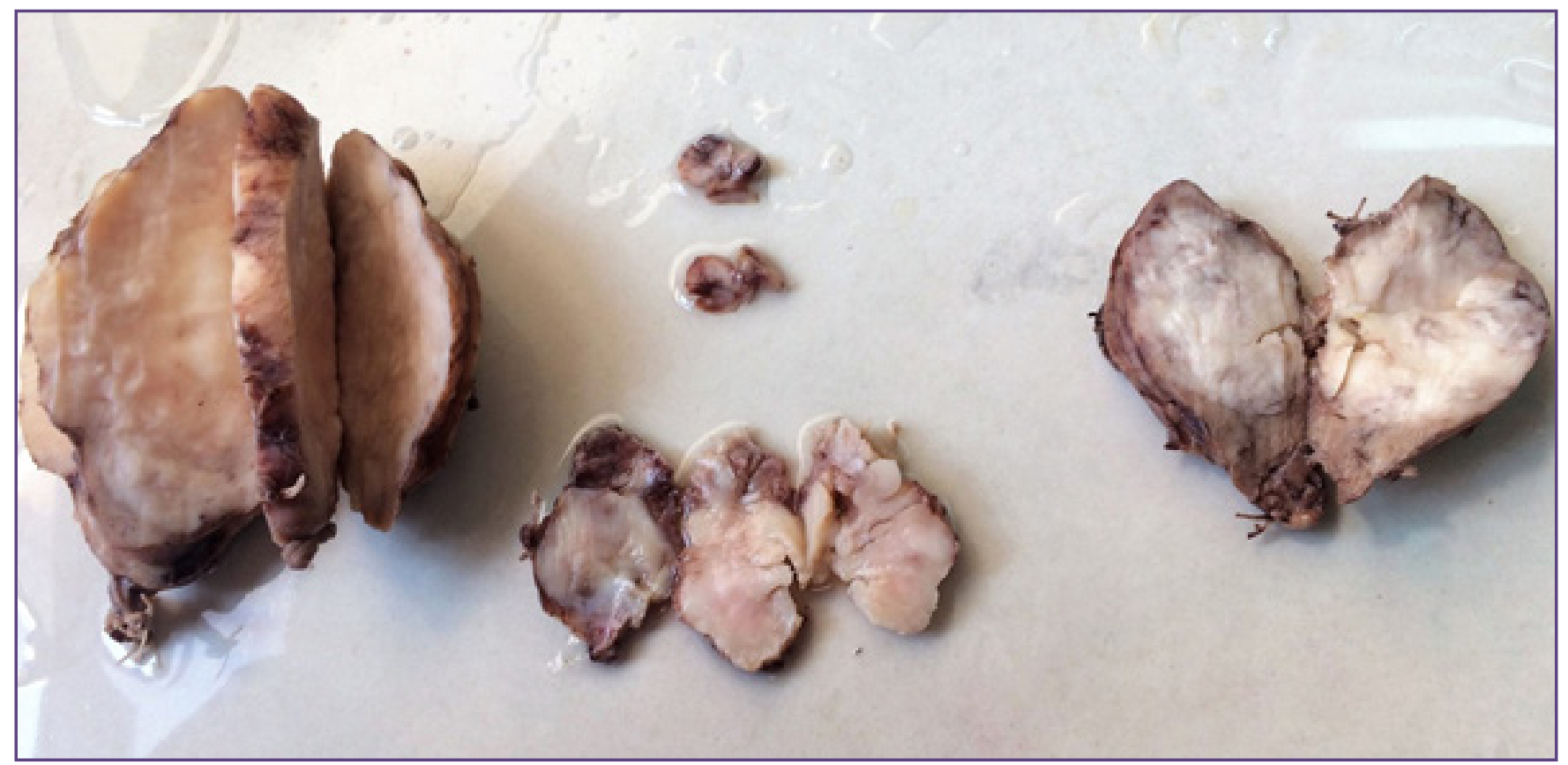

Fig. 1: Gross photograph showing grey white tumor involving the entire thyroid.

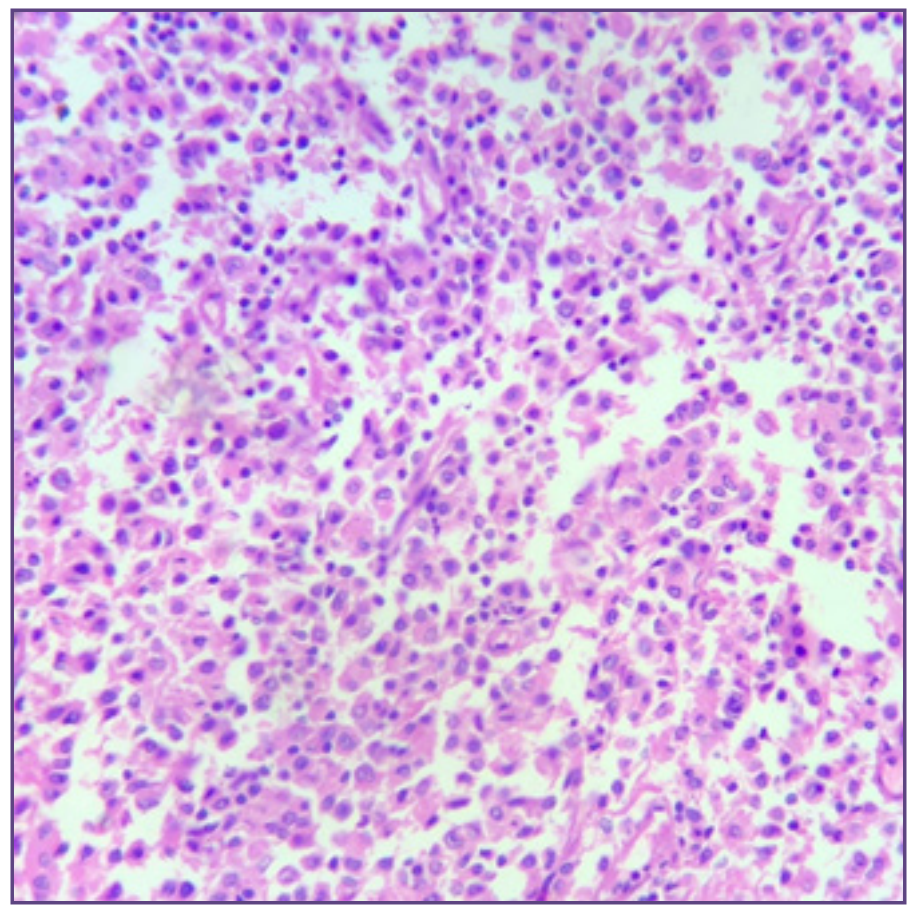

Fig. 2: H\&E, 40x, displaying malignant lymphoma cells in diffuse pattern replacing the normal thyroid. 


\section{Discussion}

Both primary and secondary lymphomas can occur in thyroid. As primary and secondary lymphomas are treated differently and prognosis varies, these need to be differentiated clearly. Primary lymphomas have better prognosis in early stage as compared to secondary lymphomas where mortality is high. ${ }^{[1]}$

It is said to be more common in post-menopausal elderly women between $60-69$ years. ${ }^{[2]}$ In our case the age of woman was 55 years. It is said that Hashimoto's thyroiditis increases the risk of developing PTL by 40$80 \%$. Lymphocytes are not normally seen in thyroid gland and therefore, the presence of lymphocytes is reportedly promoting the chances of development of PTL. ${ }^{[3]}$ The malignant transformation in Hashimoto's thyroiditis is said to occur due to chronic antigenic stimulation. ${ }^{[1]}$ Even in the present case, presence of Hashimoto's thyroiditis was seen in thyroid tissue surrounding the tumor.

The usual symptoms include painless thyroid growth over short duration along with cervical lymphadenopathy, while dyspnoea, stridor, hoarseness of voice, coughing or chocking and dysphagia may be seen due to the compression effects of tumor. ${ }^{[1]}$ Weight loss, fever and nocturnal sweats regarded as NHL symptoms are less frequently seen. ${ }^{[4]} \mathrm{In}$ our case, patient had none of these symptoms of NHL. She presented with swelling of thyroid gland and neck nodes.

The preoperative diagnostic modalities mainly include FNAC of the thyroid mass, as it is cheap, minimally invasive and gives rapid diagnosis. But PTLs may have to be differentiated from anaplastic carcinoma and Hashimoto's thyroiditis. In anaplastic carcinoma, clustering of cells along with nuclear moulding is seen with absence of lymphoglandular bodies. ${ }^{[1]}$ It is very important that anaplastic carcinoma is differentiated from DLBCL as their treatment modalities are different. DLBCL respond to chemotherapy but anaplastic carcinoma needs surgical intervention when resectable. ${ }^{[1]}$

Grossly, typical PTLs show white, homogenous fish-flesh cut surface, replacing the entire thyroid gland, as in our case. Grossly, these tumors have to be differentiated from reactive processed like Kikuchi-Fujimoto's disease or metastatic carcinoma. ${ }^{[5]}$

Microscopically, as in our case, typical DLBCLs show complete effacement of the normal thyroid architecture, replaced by diffuse large, atypical lymphoid cells, separated by fibrovascular septae. Many mitotic figures and areas of necrosis may be present. Surrounding residual thyroid tissue in our case showed features of Hashimoto's thyroiditis, proving that Hashimoto's thyroiditis may be precursor for PTLs.

For confirmatory diagnosis of DLBCL, morphology is important along with IHC markers such as CD20 and also mature B-cell markers should be positive. Also $40-50 \%$ or more of Ki-67 expression has been seen. All these markers were positive in our case. ${ }^{[5]}$

It is reported that, age more than 60 years, increased LDH and $\beta 2$ microglobulin levels and involvement of extra-nodal sites with III and IV stages of diseases are all unfavourable prognostic factors. ${ }^{[4]}$ In our case, patient is on follow up for 1 year, doing well with surgery and chemotherapy.

The histopathological differential diagnosis includes malignant melanoma, Burkitt lymphoma, small cell variant of medullary carcinoma, insular carcinoma, mediastinal Large B-cell lymphoma or plasmablastic myeloma. These can be readily being distinguished by histomorphology, IHC, Flow cytometry or genetic tests. ${ }^{[3]}$ In our case IHC confirmed our diagnosis.

\section{Conclusion}

Primary Thyroid Lymphomas of Diffuse Large B-Cell Lymphoma type are one of the important differential diagnosis of thyroid malignancies, especially in older women and correct diagnosis is important for proper treatment and prognostication.

\section{References}

1. Peixoto R, Pinto JC, Soares V, Koch P, Gomes AT. Primary thyroid lymphoma: A case report and review of the literature. Annals of Medicine and Surgery. 2017 Jan 1;13:29-33.

2. Babu KG, Lakshmaiah KC, Lokanatha D, Babu MS, Suresh TM, Rao CR, Premalata CS, Abraham LJ, Lokesh KN. Diffuse large Bcell lymphoma of the thyroid: Seven cases with review of the literature from India. Oncology, Gastroenterology and Hepatology Reports. 2015 Jul 1;4(2):81-4.

3. Rosai J, Tallini G, Thyroid Gland. In. Houston M, editor. Rosai and Ackerman's Surgical Pathology, 10th ed. United States; Elsevier Mosby; 2011. p. 535-4.

4. Vigliar E, Caleo A, Vitale M, Di Crescenzo V, Garzi A, Zeppa P. Early cytological diagnosis of extranodal stage I, primary thyroid Non-Hodgkin lymphoma in elderly patients. Report of two cases and review of the literature. BMC surgery. 2013 Oct;13(2):S49.

5. Hunt KE, Reichard KK. Diffuse large B-cell lymphoma. Archives of pathology \& laboratory medicine. 2008 Jan;132(1):118-24.

*Corresponding author:

Dr. Sudhamani S., C-24, 2-1, Pa lm Beach C.H.S., Sector - 4, Nerul, Navi Mumbai. Thane, Maharashtra. 400706., INDIA

Phone: +91 9987760804

Email: dr.sudhamani@gmail.com

Financial or other Competing Interests: None. 